\title{
Markov Modeling and Behavior Analysis of Steam Generation (SG) System in a Thermal Power Plant (TPP)
}

\author{
Vikas Modgil, \\ Department of Mechanical Engineering,DCRUST, Murthal ,Haryana, India \\ Mail id : vikashmudgil.me@dcrustm.org,vikasmodgil@yahoo.co.uk
}

\begin{abstract}
Steam generation (SG) system having five subsystems, namely Economizer, Reheater, Super heater, Furnace, Turbines, and Generator. The differential equations are acquired from the state transition diagram (STD) made pertaining to the real environment of the plant using Markov Method (MM). To get the performance of the system these equations are being work out using normalizing condition. The Performance values attained by providing the apt values of failure and repair rates (FRRs) in Markov model. Optimal Availability of the system is achieved with Genetic algorithm (GA) technique.
\end{abstract}

Keywords: Steam Generation, Electricity, Markov Method, Genetic Algorithm

\section{INTRODUCTION}

Electricity has become the most important aspect for industries in today scenario. Its consumption has increased to a huge amount due to fast development of mechanization and automation in industries. There are several ways of producing electricity such as hydroelectric plants, nuclear plant, wind mills, and thermal power plant (TPP). Out of these TPP is chief source for electricity generation. The continuous working of all the units used in TPP is very essential for generation of electricity continuously. So the availability of the total framework of thermal power plant becomes very essential. For this, the behavioral pattern of TPP has been evaluated using reliability, availability, maintainability (RAM) tools. Several researchers have contributed in the field of RAM analysis using Markov and other methods since last few decades. Dhillon \& Singh (1981) made use of the Markov Method (MM) for the steady state availability (SSA) analysis taking FRR distributed exponentially. Kumar et al., (2011) suggested an availability optimization model for a fertilizer plant using GA to cooling down the $\mathrm{CO}_{2}$ circulating within the system. Bose et al. (2012) on the basis of the Preventive Maintenance (PM) schedule of a power plant examined the RAM. The finding of the paper was that economizer was the most critical subsystem. Khanduja et al.,(2012) made a mathematical model for stock preparation in a paper industry unit using MM and also optimize the availability of the system by applying GA. Arabi et al. (2012) suggested an availability model for performance optimization SA technique. Modgil et al.,(2013) dealt with SSA model using MM applied to shoe industry and evaluated the time based system availability. Li \& Peng, (2014) evaluated the system availability and cost of operation for hybrid industrial system. Further, GA has been used as a optimizing tool. Ahmadi et al. (2019) performed the reliability evaluation of materials hauling system of the earth pressurized balance tunnel boring machine using the failure and repair data followed by the statistical techniques used for performance modeling. Malik \& Tewari, (2020) developed a mathematical model to improve performance of a coal crushing unit of thermal power plant using PSO. Kumar et al. (2020) performed SSA analysis of waste gases recovery system by applying MM. It was found that availability value is very low, and reason of this is failure of boiler tube. In the current study the SSA model has been developed for Steam Generation (SG) system in TPP. After using GA the availability optimization has been performed.

\section{SYSTEM DESCRIPTION}

Steam Generation (SG) system is the most significant system among all other systems in a TPP. The flow diagram is shown in figure1. Economizer, Reheater, Super heater, Furnace, Turbines, and Generator are the units that make up the system. 


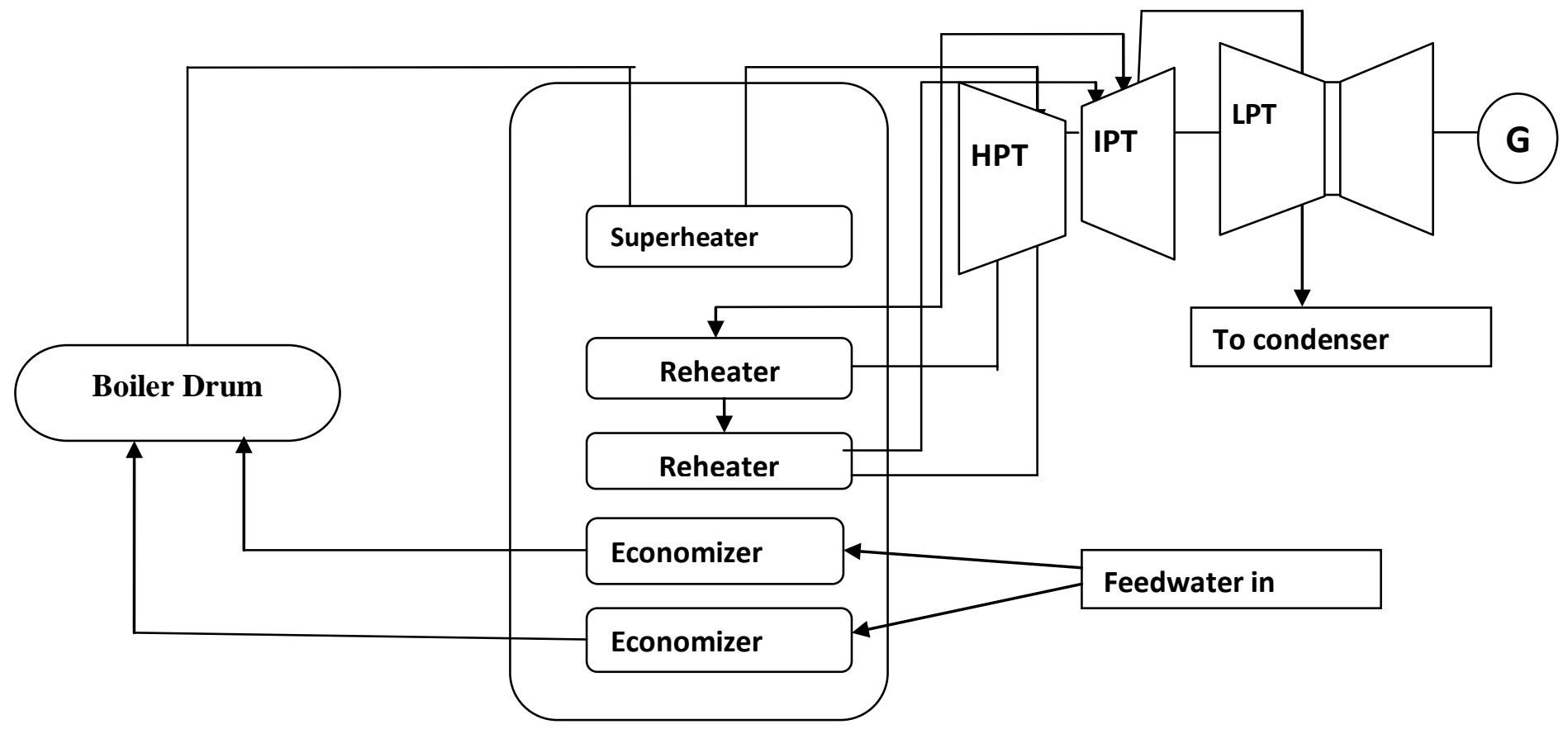

Figure 1: Flow Diagram of SG system

Economizer (E): The subsystem contains of two units subjected to minor and major failures. The inlet water to be feeded to boiler temperature is increased from $231^{\circ} \mathrm{C}$ to $280^{\circ} \mathrm{C}$ by taking the heat from flue gas in economizer. If the Economizer is partially failed then it can cause to reduction in working condition, while major failure can lead to system failure.

Super Heater (S): The subsystem contains of one unit of super heater and consists primary and final super heaters that works in series and subjected to failures. It produces superheated steam and increases its thermal energy. If the primary super heater got failed then it's working capacity gets reduced, while failures of both results to complete breakdown.

Reheater (R): The Reheater is used to heat the steam release from high pressure turbine before passing it to intermediate pressure turbine. The subsystem contains two units, failure of which causes system failure.

Turbines (T): The subsystem comprises of HP, IP and LP turbines are operating in series configuration. The breakdown of every turbine causes complete failure of system.

Generator (G): The shaft of the LPT is connected to the rotor of generator and power is transmitted to power grid system by augment the voltage. Failure of single unit results into complete failure of system.

\section{Assumptions:}

The Markov Method (MM) generally used to model the system which alters randomly and also assumption has been taken as that future state depends only on the present stage not on the previous dealings. The industrial system, generally are of these nature in which failure occurs randomly , So Markov model is best suitable to Model these systems. Here considering the following assumptions state transition diagram (STD) for the steam generation 
(SG) system had been developed as shown in figure no. 2:-

* Failure and repair rates for each subsystem are assumed to be constant and statistically independent.

* A repaired unit is as good as new, performance wise.

* Sufficient repair facilities are available.

* Service includes repairs and/or replacements.

* System may work at reduced capacity.

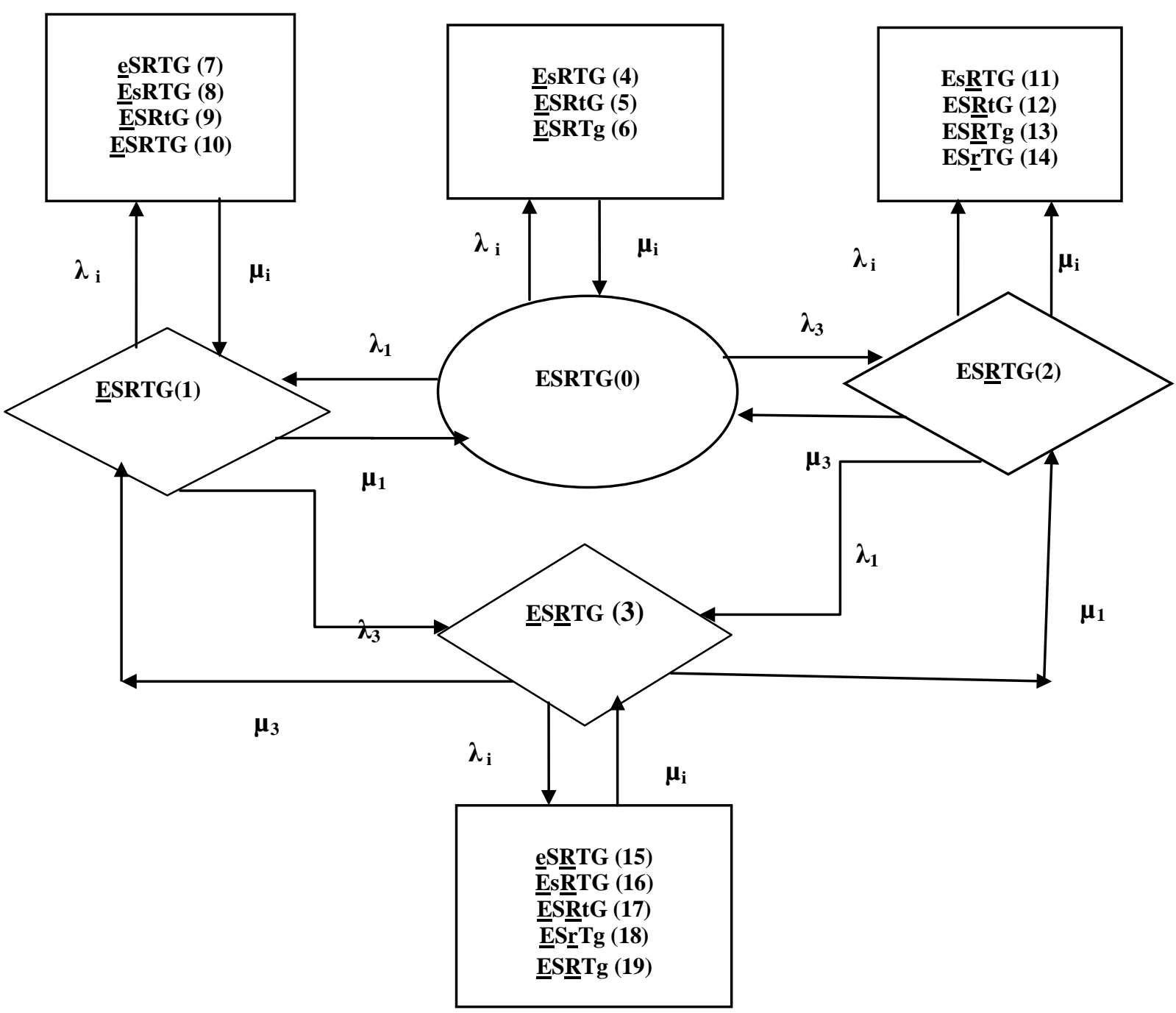

Figure 2 : State Transition Diagram (STD) for Steam Generation (SG)System 


\section{PERFORMANCE MODELING}

The various mathematical equations are derived on the basis of MM approach using STD and solved recursively to calculate SSA. The following equations related with the STD are:

$$
\begin{aligned}
& P_{0}^{\prime}(t)+\left(\lambda_{1}+\lambda_{2}+\lambda_{3}+\lambda_{4}+\lambda_{5}\right) P_{0}(\mathrm{t})=\mu_{1} P_{2}(\mathrm{t})+\mu_{3} P_{2}(\mathrm{t})+\mu_{2} \lambda_{4}(\mathrm{t})+\mu_{4} \lambda_{5}(\mathrm{t})+\mu_{5} \lambda_{6}(\mathrm{t}) \\
& P_{1}^{\prime}(t)+\left(\mu_{1}+\lambda_{3}+\lambda_{2}+\lambda_{6}+\lambda_{4}+\lambda_{5}\right) P_{1}(t)=\lambda_{1} P_{0}(t)+\mu_{3} P_{3}(t)+\mu_{6} P_{7}(t)+\mu_{2} P_{8}(t)+\mu_{4} P_{19}(t)+ \\
& \mu_{5} P_{10}(t) \\
& P_{2}^{\prime}(t)+\left(\mu_{3}+\lambda_{1}+\lambda_{2}+\lambda_{6}+\lambda_{4}+\lambda_{5}\right) P_{2}(t)=\mu_{1} P_{3}(t)+\lambda_{3} P_{0}(t)+\mu_{2} P_{11}(t)+\mu_{4} P_{12}(t)+\mu_{5} P_{13}(t)+ \\
& \mu_{7} P_{14}(t) \\
& P_{3}^{\prime}(t)+\left(\mu_{3}+\mu_{1}+\lambda_{6}+\lambda_{2}+\lambda_{4}+\lambda_{5}+\lambda_{7}\right) P_{3}(t)=\lambda_{3} P_{1}(t)+\lambda_{1} P_{2}(t)+\mu_{6} P_{15}(t)+\mu_{2} P_{16}(t)+ \\
& \mu_{7} P_{17}(t)+\mu_{4} P_{18}(t)+\mu_{5} P_{19}(t) \\
& P_{4}^{\prime}(t)+\mu_{2} P_{4}(t)=\lambda_{2} P_{0}(t) \\
& P_{5}^{\prime}(t)+\mu_{4} P_{5}(t)=\lambda_{4} P_{0}(t) \\
& P_{6}^{\prime}(t)+\mu_{5} P_{6}(t)=\lambda_{5} P_{0}(t) \\
& P_{7}^{\prime}(t)+\mu_{6} P_{7}(t)=\lambda_{6} P_{1}(t) \\
& P_{8}^{\prime}(t)+\mu_{2} P_{8}(t)=\lambda_{2} P_{1}(t) \\
& P_{9}^{\prime}(t)+\mu_{4} P_{9}(t)=\lambda_{4} P_{1}(t) \\
& P_{10}^{\prime}(t)+\mu_{5} P_{10}(t)=\lambda_{5} P_{1}(t) \\
& P_{11}^{\prime}(t)+\mu_{2} P_{11}(t)=\lambda_{2} P_{2}(t) \\
& P_{12}^{\prime}(t)+\mu_{4} P_{12}(t)=\lambda_{4} P_{2}(t) \\
& P_{13}^{\prime}(t)+\mu_{5} P_{13}(t)=\lambda_{5} P_{2}(t) \\
& P_{14}^{\prime}(t)+\mu_{7} P_{14}(t)=\lambda_{7} P_{2}(t) \\
& P_{15}^{\prime}(t)+\mu_{6} P_{15}(t)=\lambda_{6} P_{3}(t) \\
& P_{16}^{\prime}(t)+\mu_{2} P_{16}(t)=\lambda_{2} P_{3}(t) \\
& P_{17}^{\prime}(t)+\mu_{7} P_{17}(t)=\lambda_{7} P_{3}(t) \\
& P_{18}^{\prime}(t)+\mu_{4} P_{18}(t)=\lambda_{4} P_{3}(t) \\
& P_{19}^{\prime}(t)+\mu_{5} P_{19}(t)=\lambda_{5} P_{3}(t)
\end{aligned}
$$

Using initial condition at $\mathrm{t}=0, \mathrm{P}_{\mathrm{i}}(\mathrm{t})=1$ for $\mathrm{i}=0$ otherwise $\mathrm{P}_{\mathrm{i}}(\mathrm{t})=0$ for steady state availability , the system is studied $\mathrm{d} / \mathrm{dt} \rightarrow 0$ and $\mathrm{t} \rightarrow 0$.

$$
\begin{aligned}
& P_{0}^{\prime}+\left(\lambda_{1}+\lambda_{2}+\lambda_{3}+\lambda_{4}+\lambda_{5}\right) P_{0}=\mu_{1} P_{2}+\mu_{3} P_{2}+\mu_{2} \lambda_{4}+\mu_{4} \lambda_{5}+\mu_{5} \lambda_{6} \\
& P_{1}^{\prime}+\left(\mu_{1}+\lambda_{3}+\lambda_{2}+\lambda_{6}+\lambda_{4}+\lambda_{5}\right) P_{1}=\lambda_{1} P_{0}+\mu_{3} P_{3}+\mu_{6} P_{7}+\mu_{2} P_{8}+\mu_{4} P_{19}+\mu_{5} P_{10} \\
& P_{2}^{\prime}+\left(\mu_{3}+\lambda_{1}+\lambda_{2}+\lambda_{6}+\lambda_{4}+\lambda_{5}\right) P_{2}=\mu_{1} P_{3}+\lambda_{3} P_{0}+\mu_{2} P_{11}+\mu_{4} P_{12}+\mu_{5} P_{13}+\mu_{7} P_{14} \\
& P_{3}^{\prime}+\left(\mu_{3}+\mu_{1}+\lambda_{6}+\lambda_{2}+\lambda_{4}+\lambda_{5}+\lambda_{7}\right) P_{3}=\lambda_{3} P_{1}+\lambda_{1} P_{2}+\mu_{6} P_{15}+\mu_{2} P_{16}+\mu_{7} P_{17}+\mu_{4} P_{18}+\mu_{5} P_{19} \\
& P_{4}^{\prime}+\mu_{2} P_{4}=\lambda_{2} P_{0} \\
& P_{5}^{\prime}+\mu_{4} P_{5}=\lambda_{4} P_{0} \\
& P_{6}^{\prime}+\mu_{5} P_{6}=\lambda_{5} P_{0} \\
& P_{7}^{\prime}+\mu_{6} P_{7}=\lambda_{6} P_{1} \\
& P_{8}^{\prime}+\mu_{2} P_{8}=\lambda_{2} P_{1} \\
& P_{9}^{\prime}+\mu_{4} P_{9}=\lambda_{4} P_{1} \\
& P_{10}^{\prime}+\mu_{5} P_{10}=\lambda_{5} P_{1}
\end{aligned}
$$


$P_{11}^{\prime}+\mu_{2} P_{11}=\lambda_{2} P_{2}$

$P_{12}^{\prime}+\mu_{4} P_{12}=\lambda_{4} P_{2}$

$P_{13}^{\prime}+\mu_{5} P_{13}=\lambda_{5} P_{2}$

$P_{14}^{\prime}+\mu_{7} P_{14}=\lambda_{7} P_{2}$

$P_{15}^{\prime}+\mu_{6} P_{15}=\lambda_{6} P_{3}$

$P_{16}^{\prime}+\mu_{2} P_{16}=\lambda_{2} P_{3}$

$P_{17}^{\prime}+\mu_{7} P_{17}=\lambda_{7} P_{3}$

$P_{18}^{\prime}+\mu_{4} P_{18}=\lambda_{4} P_{3}$

$P_{19}^{\prime}+\mu_{5} P_{19}=\lambda_{5} P_{3}$

Initially it is assumed that initially all the subsystems are in working state, therefore the sum of total probable state is one. Solving the above equations, we get the steady-state availability (SSA) for the model for the SG system of TPP :-

$P_{0}+P_{1}+P_{2}+\ldots \ldots . .+P_{19}=1$

$S S A=A_{V}=\left[P_{0}+P_{1}+P_{2}+P_{3}\right]$

To find the availability, the subsequent input values of the FRR for the system chosen are given, and using these availability for each component of SG system has been evaluated and its various levels are revealed in table 1 to 5 .

Table 1 show the effect of economizer's FRR on system availability. It shows that with varying economizer failure rate (FR) from 0.9249 to 0.8570 , availability diminishes by about $7.3 \%$. In same way, as repair rates (RR) increase from 0.9249 to 0.9553 , system availability increases by $3.2 \%$.

Table 2 reveals the effect of super heater FRR on system availability. With change in super heater's FR from 0.9249 to 0.9115 , availability lessen by about $1.4 \%$. Also, as super heater RR increase from 0.9249 to 0.9284 , availability rises by $0.3 \%$.

Table 3 shows that with decreasing reheater FR from 0.9249 to 0.9021 , availability lessen by about $2.4 \%$. likewise, as RR increase from 0.9249 to 0.9335 , system availability augmented by $0.9 \%$.

Table 4 reveals that with decreasing turbine FR from 0.9249 to 0.9203 , availability decline by about $0.4 \%$. Similarly, as RR increase from 0.9249 to 0.9258 , it has been enhanced by $0.09 \%$.

Table 5 shows that with decreasing generator FR from 0.9249 to 0.8939 , availability declines by 3.3\%. Similarly, as RR increase from 0.9249 to 09330 , availability go up by $0.8 \%$.

Table 1 Behavior Pattern for change in FRR for Economizer

\begin{tabular}{|l|l|l|l|l|}
\hline$\mu_{1}$ & & & & \\
\hline
\end{tabular}


Table 2 Behavior Pattern for change in FRR for Superheater

\begin{tabular}{|l|l|l|l|l|}
\hline & 0.0008 & 0.0016 & 0.0024 & 0.0032 \\
\hline 0.15 & 0.9249 & 0.9204 & 0.9159 & 0.9115 \\
\hline 0.30 & 0.9272 & 0.9249 & 0.9227 & 0.9204 \\
\hline 0.45 & 0.9280 & 0.9265 & 0.9249 & 0.9234 \\
\hline 0.60 & 0.9284 & 0.9272 & 0.9261 & 0.9249 \\
\hline
\end{tabular}

Table 3 : Behavior Pattern for change in FRR for Reheater

\begin{tabular}{|l|l|l|l|l|}
\hline & 0.01 & 0.02 & 0.03 & 0.04 \\
\hline 0.08 & & & & \\
\hline 0.16 & 0.9249 & 0.9157 & 0.9082 & 0.9021 \\
\hline 0.24 & 0.9305 & 0.9249 & 0.9200 & 0.9157 \\
\hline 0.32 & 0.9325 & 0.9286 & 0.9249 & 0.9216 \\
\hline
\end{tabular}

Table 4 Behavior Pattern for change in FRR for Turbine

\begin{tabular}{|c|c|c|c|c|}
\hline$\lambda_{4}$ & 0.0005 & 0.0010 & 0.0015 & 0.0020 \\
\hline 0.25 & 0.9249 & 0.9234 & 0.9219 & 0.9203 \\
\hline 0.35 & 0.9254 & 0.9243 & 0.9232 & 0.9221 \\
\hline 0.45 & 0.9256 & 0.9248 & 0.9239 & 0.9231 \\
\hline 0.55 & $\begin{array}{l}0.9258 \\
\end{array}$ & 0.9251 & 0.9244 & 0.9237 \\
\hline
\end{tabular}


Table 5 Behavior Pattern for change in FRR for Generator

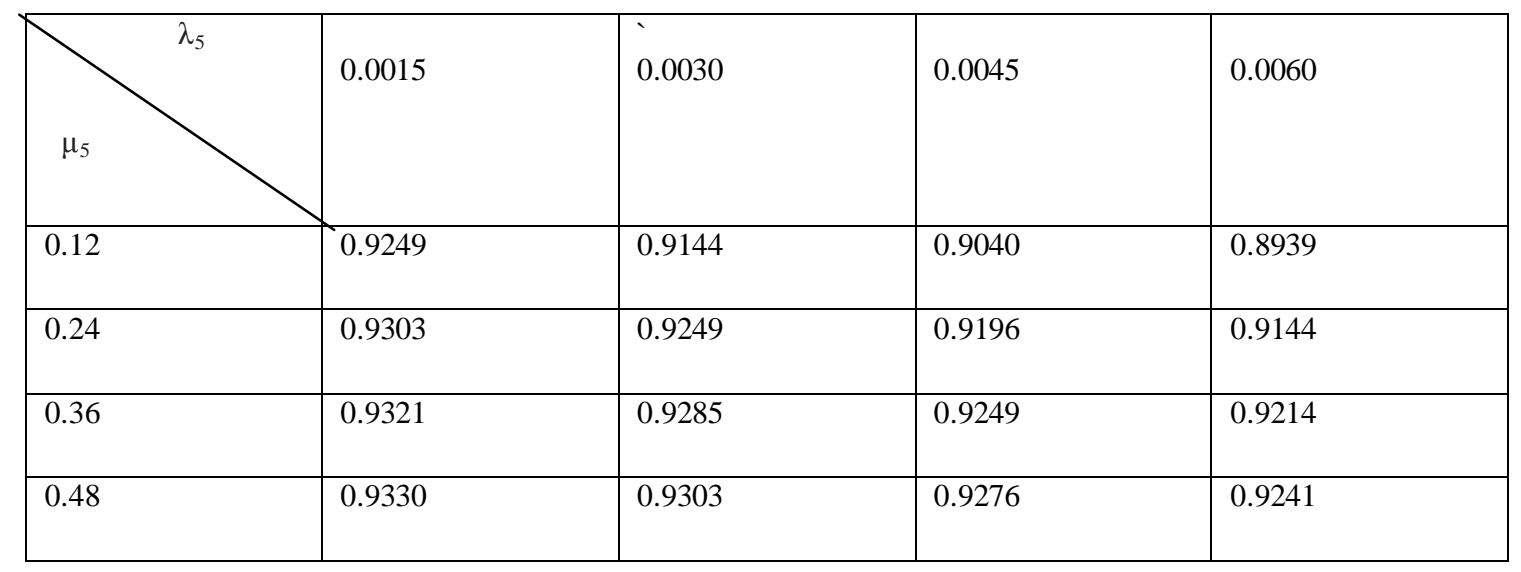

Availability levels as given in Tables 1 to 5 demonstrate variation in system availability with change in FRR for different subsystems. The results of the study reflect that Economizer has highest effect on system performance; by changing its RR performance increases by 3.2\%. Likewise, the turbine has the least effect.

The maintenance priorities for various subsystems must follow the following order:

- Economizer

- Generator

- Reheater

- Superheater

- Turbine

\section{AVAILABILITY OPTIMIZATION FOR STEAM GENERATION (SG) SYSTEM USING GENETIC ALGORITHM (GA)}

Genetic Algorithm (GA) is a search-based optimization method based on the philosophy of Genetics and Natural Selection. It is exercised to find optimal solutions to find the required objective (here, Maximum system performance i.e. utmost availability). Various parameters on which system performance depends are coded in real structures and are mapped amid their lower and upper bounds Here, Firstly the optimization is done by varying the generation size from 50 to 450 in step of 50 as shown in Table 2.

As revealed from table, the optimal availability of SG system obtained as $92.797 \%$ at 100 number of generation. The corresponding values of FRR are $\lambda_{1}=0.03417, \mu_{1}=0.06, \lambda_{2}=0.00319, \mu_{2}=0.15, \lambda_{3}=0.01078, \mu_{3}=0.32 \lambda_{4}=$ 0.00199, $\mu_{4}=0.2540 \lambda_{5}=0.006, \mu_{5}=0.1227$, which is the finest potential combination of FRR of different subsystems. 
Table 6: Optimal Availability of SG System by varying Generation size

\begin{tabular}{|c|l|l|l|l|l|l|l|l|l|l|}
\hline $\begin{array}{r}\text { Number of } \\
\text { Generation }\end{array}$ & Availability $\lambda_{1}$ & $\lambda_{2}$ & $\lambda_{3}$ & $\lambda_{4}$ & $\lambda_{5}$ & $\mu_{1}$ & $\mu_{2}$ & $\mu_{3}$ & $\mu_{4}$ & $\mu_{5}$ \\
\hline 500.92697 & 0.04 & 0.00324 & 0.0175 & 0.002 & 0.006 & 0.06 & 0.151 & 0.3013 & 0.25 & 0.12 \\
\hline $\mathbf{1 0 0 0 . 9 2 7 9 7}$ & $\mathbf{0 . 0 3 4 1 7}$ & $\mathbf{0 . 0 0 3 1 9}$ & $\mathbf{0 . 0 1 0 7 8}$ & $\mathbf{0 . 0 0 1 9 9}$ & $\mathbf{0 . 0 0 5 9 9}$ & $\mathbf{0 . 0 6 4 6}$ & $\mathbf{0 . 1 5 1 1}$ & $\mathbf{0 . 3 1 9 9}$ & $\mathbf{0 . 2 5 4 0}$ & $\mathbf{0 . 1 2 2 7}$ \\
\hline 1500.92669 & 0.04 & 0.0032 & 0.01002 & 0.002 & 0.006 & 0.06 & 0.15 & 0.31 & 0.2501 & 0.12 \\
\hline 2000.92667 & 0.04 & 0.0032 & 0.01087 & 0.002 & 0.006 & 0.06 & 0.15001 & 0.32 & 0.2525 & 0.12001 \\
\hline 2500.92662 & 0.04 & 0.0032 & 0.01 & 0.002 & 0.006 & 0.06004 & 0.1501 & 0.32 & 0.25 & 0.12 \\
\hline 3000.92659 & 0.04 & 0.0032 & 0.0101 & 0.002 & 0.006 & 0.06344 & 0.15 & 0.32 & 0.25 & 0.12 \\
\hline 3500.92658 & 0.04 & 0.0032 & 0.01008 & 0.002 & 0.006 & 0.06006 & 0.15001 & 0.32 & 0.25 & 0.12 \\
\hline 4000.92657 & 0.04 & 0.0032 & 0.01 & 0.002 & 0.006 & 0.06 & 0.15003 & 0.32 & 0.25 & 0.12 \\
\hline 4500.92657 & 0.04 & 0.0032 & 0.01 & 0.002 & 0.006 & 0.06 & 0.15003 & 0.32 & 0.25 & 0.12 \\
\hline
\end{tabular}

Secondly, the optimization is completed by increasing the population size starting as 20 and ends at 100 in step of 10, as shown in Table 7. It has been observed that the optimal performance of SG system comes out to be 93.067\% at 40 number of generation. The best possible combination of FRR are $\lambda_{1}=0.04, \mu_{1}=0.06, \lambda_{2}=0.0032$, $\mu_{2}=0.19, \lambda_{3}=0.01005, \mu_{3}=0.33 \lambda_{4}=0.002, \mu_{4}=0.25 \lambda_{5}=0.006, \mu_{5}=0.12$, which is the most probable combination of FRR of different components.

Table 7: Optimal Availability of SG System by varying Population size

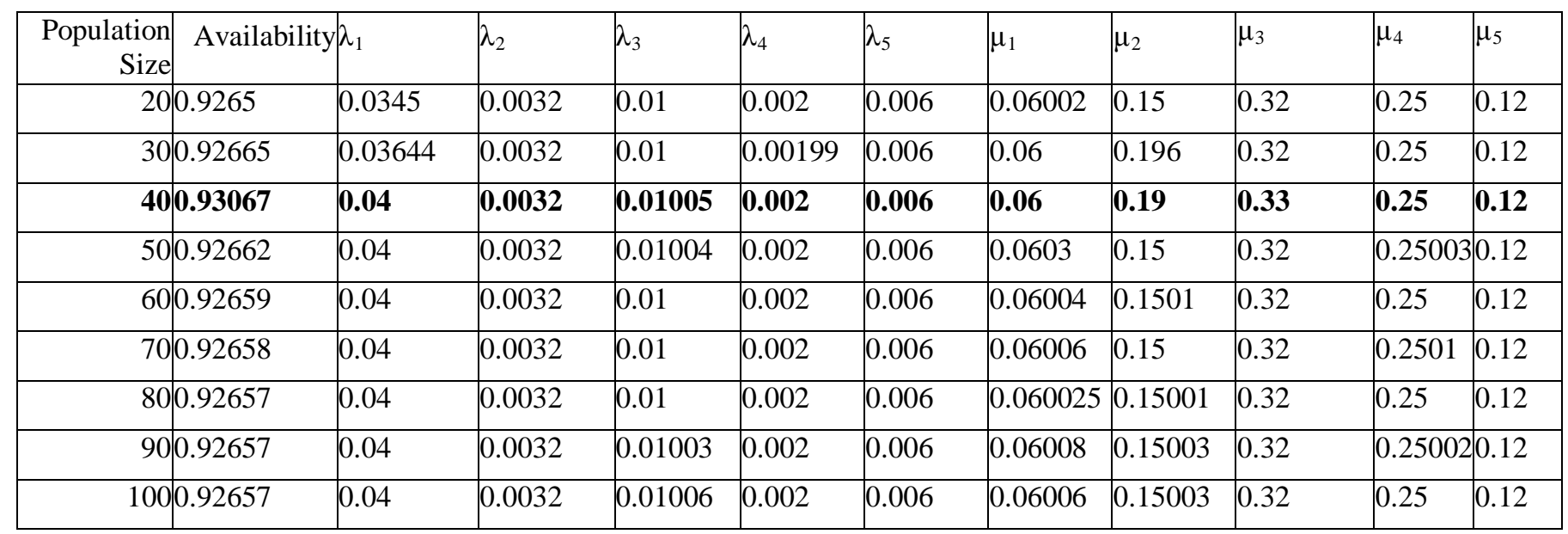

\section{CONCLUSIONS}

The SSA of SG system is obtained as $92.49 \%$ by considering FRR gathered from industry history sheets. GA is used to get the optimal availability value which comes as $93.067 \%$ which is approximately enhancement of $0.62 \%$ as shown on table 6. 
Table 4: SSA and Optimal Availability Using G.A.

\begin{tabular}{|c|c|c|}
\hline System & SSA & Optimal Availability (Considering GA) \\
\hline Steam & $92.49 \%$ & $93.067 \%$ \\
Generation & $\lambda_{1}=0.04, \mu_{1}=0.06, \lambda_{2}=0.0008, \mu_{2}=$ & $\lambda_{1}=0.03417, \mu_{1}=0.06, \lambda_{2}=0.00319, \mu_{2}=0.15, \lambda_{3}=$ \\
system & $0.15, \lambda_{3}=0.01, \mu_{3}=0.32 \lambda_{4}=0.002$, \\
& $\mu_{4}=0.25 \lambda_{5}=0.006, \mu_{5}=0.12$ & $0.01078, \mu_{3}=0.32 \lambda_{4}=0.00199, \mu_{4}=0.2540 \lambda_{5}=0.006, \mu_{5}=$ \\
& & 0.1227 \\
\hline
\end{tabular}

To acquire the optimum availability level, the consequent FRR of the subsystems should be maintained. These results can help the industrial management to take better decision for enhancing the SG system's performance.

\section{REFERENCES}

Ahmadi, S., Moosazadeh, S., Hajihassani, M.,Moomivand, H. \& Rajaei, M. "Reliability, availability and maintainability analysis of the conveyor system in mechanized tunneling” Measurement, 145, (2019), pp 756-764.

Arabi, A.A.Y. \&Jahromi, A.E. "Developing a new model for availability of a series repairable system with multiple cold-standby subsystems and optimization using simulated annealing considering redundancy and repair facility allocation”, International Journal of System Assurance Engineering and Management, Vol. 3,No. 4, 2012, pp 310-322.

Bose, D., Chattopadhyay, S., Bose, G., Adhikary, D., \&Mitra, S. (2012), "RAM investigation of coal fired thermal power plants: a case study”, International Journal of Industrial Engineering Computations, Vol. 3(3), 2012, pp 423-434.

Dhillon, B. S., \& Singh, C. “Engineering reliability: new techniques and applications”, Wiley Publishers (1981).

Kumar, P. and Tewari, P.C. , "Performance analysis and optimization for CSDGB filling system of a beverage plant using particle swarm optimization. International Journal of Industrial Engineering Computation”, 2020, Vol. 8No. (3), pp 303-314.

Kumar, S. \&Tewari, P. C. (2011), "Mathematical modeling and performance optimization of $\mathrm{CO}_{2}$ cooling system of a fertilizer plant”, International Journal of Industrial Engineering Computation,2011,Vol. 2(3),pp 689-698.

Khanduja, R., Tewari, P. C. \& Chauhan, R. S., "Performance modeling and optimization for the stock preparation unit of a paper plant using genetic algorithm” International Journal of Quality Reliability and Management, 2012 vol. 28(6), pp 688-703.

Kumar, R., Sharma, A., \&Tewari, "Markov approach to evaluate the availability simulation model for power generation system in a thermal power plant” International Journal of Industrial Engineering Computations, Vol. 3(5), 2012, pp 743-750.

Li, Y. F., \& Peng, R. Availability modeling and optimization of dynamic multi-state series-parallel systems with random reconfiguration. Reliability Engineering \& System Safety, 127, 2014, 47-57.

Malik S. \& Tewari, P. C. "Optimization of coal handling system Performability for a thermal power plant using PSO algorithm” International Journal of Quality \& Reliability Management, 10(3), 2020, pp 359-376. 